PROCEEDINGS OF THE

AMERICAN MATHEMATICAL SOCIETY

Volume 134, Number 9, September 2006, Pages 2685-2694

S 0002-9939(06)08316-X

Article electronically published on March 23, 2006

\title{
LINEAR INDEPENDENCE OF PSEUDO-SPLINES
}

\author{
BIN DONG AND ZUOWEI SHEN \\ (Communicated by David R. Larson)
}

\begin{abstract}
In this paper, we show that the shifts of a pseudo-spline are linearly independent. This is stronger than the (more obvious) statement that the shifts of a pseudo-spline form a Riesz system. In fact, the linear independence of a compactly supported (refinable) function and its shifts has been studied in several areas of approximation and wavelet theory. Furthermore, the linear independence of the shifts of a pseudo-spline is a necessary and sufficient condition for the existence of a compactly supported function whose shifts form a biorthogonal dual system of the shifts of the pseudo-spline.
\end{abstract}

\section{INTRODUCTION}

A pseudo-spline is a compactly supported refinable function as was first introduced in 8. This paper is to prove that the shifts (integer translations) of a pseudo-spline are linearly independent.

A compactly supported function $\phi \in L_{2}(\mathbb{R})$ is refinable if it satisfies the refinement equation

$$
\phi=2 \sum_{k \in \mathbb{Z}} a(k) \phi(2 \cdot-k),
$$

where $a$, called the refinement mask of $\phi$, is a finitely supported sequence.

By $L_{2}(\mathbb{R})$ we denote all the functions $f(x)$ satisfying

$$
\|f(x)\|_{L_{2}(\mathbb{R})}:=\left(\int_{\mathbb{R}}|f(x)|^{2} d x\right)^{\frac{1}{2}}<\infty,
$$

and by $\ell_{2}(\mathbb{Z})$ the set of all sequences $c$ defined on $\mathbb{Z}$ such that

$$
\|c\|_{\ell_{2}(\mathbb{Z})}:=\left(\sum_{j \in \mathbb{Z}}|c(j)|^{2}\right)^{\frac{1}{2}}<\infty .
$$

The Fourier-Laplace transform of a compactly supported (measurable) function $f$ is defined by

$$
\widehat{f}(\zeta):=\int_{\mathbb{R}} f(t) e^{-i \zeta t} d t, \quad \zeta \in \mathbb{C} .
$$

Received by the editors September 22, 2004 and, in revised form, April 6, 2005.

2000 Mathematics Subject Classification. Primary 42C40, 41A30.

Key words and phrases. Linear independence, pseudo-spline, stability.

This research was supported by several grants from the Department of Mathematics, National University of Singapore.

(C)2006 American Mathematical Society Reverts to public domain 28 years from publication 
When $f$ is compactly supported and bounded, the Fourier-Laplace transform of $f$ is analytic. When $\zeta$ is restricted to $\mathbb{R}, \widehat{f}$ becomes the Fourier transform of $f$.

For a given finitely supported sequence $c$, its corresponding Laurent polynomial is defined by

$$
\tilde{c}(z):=\sum_{j \in \mathbb{Z}} c(j) z^{j}, \quad \text { for } z \in \mathbb{C} \backslash\{0\} .
$$

The corresponding trigonometric polynomial is

$$
\widehat{c}(\xi)=\tilde{c}\left(e^{-i \xi}\right), \quad \xi \in \mathbb{R} .
$$

With these, the refinement equation (1.1) can be written in terms of its Fourier transform as

$$
\widehat{\phi}(\xi)=\widehat{a}(\xi / 2) \widehat{\phi}(\xi / 2), \quad \xi \in \mathbb{R} .
$$

We also call $\widehat{a}$ the refinement mask of $\phi$ for convenience.

The refinement equation (1.1) can also be written in terms of its Fourier-Laplace transform as

$$
\widehat{\phi}(\zeta)=\tilde{a}\left(e^{-i \zeta / 2}\right) \widehat{\phi}(\zeta / 2), \quad \text { for all } \zeta \in \mathbb{C} .
$$

We call $\tilde{a}$ a symbol of $\phi$.

Pseudo-splines were first introduced in 8 and a comprehensive study, especially the regularity analysis, was given in 11]. A pseudo-spline is a compactly supported refinable function defined by its finitely supported refinement mask. There are two types of pseudo-splines. The refinement mask of a pseudo-spline of type I with order $(m, l)$ (see [8]) is given by

$$
\left.{ }_{1} \widehat{a}(\xi)\right|^{2}:=\left.{ }_{1} \widehat{a}_{(m, l)}(\xi)\right|^{2}:=\cos ^{2 m}(\xi / 2) \sum_{j=0}^{l}\left(\begin{array}{c}
m+l \\
j
\end{array}\right) \sin ^{2 j}(\xi / 2) \cos ^{2(l-j)}(\xi / 2),
$$

and type II with order $(m, l)$ (see [11]) is given by

$$
{ }_{2} \widehat{a}(\xi):={ }_{2} \widehat{a}_{(m, l)}(\xi):=\cos ^{2 m}(\xi / 2) \sum_{j=0}^{l}\left(\begin{array}{c}
m+l \\
j
\end{array}\right) \sin ^{2 j}(\xi / 2) \cos ^{2(l-j)}(\xi / 2) .
$$

It is clear that $\left|{ }_{1} \widehat{a}_{(m, l)}(\xi)\right|^{2}={ }_{2} \widehat{a}_{(m, l)}(\xi)$. Furthermore, ${ }_{1} \widehat{a}_{(m, l)}(\xi)$ is a $2 \pi$-periodic trigonometric polynomial with real coefficients by Féjer-Riesz lemma (see e.g. Lemma 6.1 .3 of $[7]$ ).

The corresponding pseudo-splines can be defined in terms of their Fourier transforms as

$$
{ }_{k} \widehat{\phi}(\xi):={ }_{k} \widehat{\phi}_{(m, l)}(\xi):=\prod_{j=1}^{\infty}{ }_{k} \widehat{a}_{(m, l)}\left(2^{-j} \xi\right), k=1,2,
$$

with ${ }_{k} \widehat{\phi}_{(m, l)}(0)=1$. Unless it is necessary, we use ${ }_{k} a$ and ${ }_{k} \phi$ instead of ${ }_{k} a_{(m, l)}$ and ${ }_{k} \phi_{(m, l)}, k=1,2$, i.e. we always drop the subscript " $(m, l)$ " in ${ }_{k} a_{(m, l)}$ and ${ }_{k} \phi_{(m, l)}$ for simplicity.

The regularity analysis of the pseudo-spline was given in [11. It was shown that pseudo-splines with order $(m, l), m \geq 2$, have positive regularity exponents. Hence, all pseudo-splines are bounded and in $L_{2}(\mathbb{R})$ (see Proposition 3.5 of [11]). The regularity exponent of a pseudo-spline is estimated in terms of its mask and depends on its order $(m, l)$. Interested readers should consult [1] for the details. 
Pseudo-splines consist of a rich family of compactly supported refinable functions. Together with the unitary extension principle of [21], they give a wide range of choices of wavelet systems that balance and meet various demands, such as the support and regularity of wavelets, and the approximation power of the truncated tight frame series (see e.g. [8]), in time-frequency analysis. When $l=0$, pseudosplines of both types are B-splines. Recall that a B-spline (see e.g. [1]) with order $m$ and its refinement mask are defined by

$$
\widehat{B}_{m}(\xi)=e^{-i j \frac{\xi}{2}}\left(\frac{\sin (\xi / 2)}{\xi / 2}\right)^{m} \text { and } \widehat{a}(\xi)=e^{-i j \frac{\xi}{2}} \cos ^{m}(\xi / 2),
$$

where $j=0$ when $m$ is even, $j=1$ when $m$ is odd. When $l=m-1$, pseudo-splines of type I are the orthogonal refinable functions (i.e. the refinable functions with orthonormal shifts which were constructed by Daubechies in [7]). Finally, pseudosplines of type II with order $(m, m-1)$ are the interpolatory refinable functions (which were first studied by Dubuc in [12]). A continuous function $\phi$ is said to be interpolatory if

$$
\phi(j)=\delta(j), \quad j \in \mathbb{Z},
$$

where $\delta(0)=1$ and $\delta(j)=0$, for $j \neq 0$.

For a compactly supported function $\phi \in L_{2}(\mathbb{R})$ and some sequence $b \in \ell(\mathbb{Z})$, where $\ell(\mathbb{Z})$ denotes the space of all complex-valued sequences defined on $\mathbb{Z}$, the semi-convolution of $\phi$ and $b$ is defined by

$$
\phi *^{\prime} b:=\sum_{j \in \mathbb{Z}} b(j) \phi(\cdot-j) .
$$

Note that for any $b \in \ell(\mathbb{Z})$ and a compactly supported function $\phi \in L_{2}(\mathbb{R}), \phi *^{\prime} b$ converges uniformly on any compact set (see e.g. [3]).

In order to introduce the concept of the linear independence of the shifts of a compactly supported function $\phi$, we first recall the notion of stability of $\phi$ which is related to, somehow weaker than, the linear independence. A function $\phi \in L_{2}(\mathbb{R})$ is stable if there exist $0<C_{1}, C_{2}<\infty$, such that for any sequence $b \in \ell_{2}(\mathbb{Z})$,

$$
C_{1}\|b\|_{\ell_{2}(\mathbb{Z})} \leq\left\|\phi *^{\prime} b\right\|_{L_{2}(\mathbb{R})} \leq C_{2}\|b\|_{\ell_{2}(\mathbb{Z})} .
$$

The stability of function $\phi \in L_{2}(\mathbb{R})$ can also be characterized by its bracket product (see e.g. 3] and [15]). Recall that the bracket product of $L_{2}(\mathbb{R})$ functions $f$ and $g$ is defined by

$$
[\widehat{f}, \widehat{g}](\xi):=\sum_{k \in \mathbb{Z}} \widehat{f}(\xi+2 \pi k) \overline{\widehat{g}(\xi+2 \pi k)} .
$$

It is also well known that (see e.g. [3], 7], [15] and [20]) a function $\phi \in L_{2}(\mathbb{R})$ is stable if and only if there exist two constants $0<C_{1}, C_{2}<\infty$ such that

$$
C_{1} \leq[\widehat{\phi}, \widehat{\phi}](\xi) \leq C_{2}
$$

holds for almost every $\xi \in \mathbb{R}$.

When $\phi$ is compactly supported in $L_{2}(\mathbb{R})$, it was shown by Jia and Micchelli in Theorem 2.1 of [15] that the upper bound of (1.6) always holds. Furthermore, Theorem 3.5 of [15] asserts that the lower bound of (1.6) is equivalent to

$$
(\widehat{\phi}(\xi+2 \pi k))_{k \in \mathbb{Z}} \neq \mathbf{0} \text { for all } \xi \in \mathbb{R}
$$

where $\mathbf{0}$ denotes the zero sequence in $\ell(\mathbb{Z})$. Hence, Jia and Micchelli proved that the stability of a compactly supported function $\phi \in L_{2}(\mathbb{R})$ is equivalent to (1.8). 
A compactly supported function $\phi \in L_{2}(\mathbb{R})$ and its shifts are linearly independent if, for $b \in \ell(\mathbb{Z})$,

$$
\phi *^{\prime} b=0 \text { implies } b(j)=0, \text { for all } j \in \mathbb{Z} .
$$

The linear independence of a compactly supported function was first studied by Dahmen and Micchelli in 9] and 10, and Jia in 13 and 14 in the context of box splines. In 19] Ron (also see 4]) studied the linear independence of compactly supported distributions in terms of their Fourier-Laplace transforms. Applying Proposition 2.1 of [19] (also see [16]), one obtains that for an arbitrary compactly supported single variable distribution, which is not identically zero, there are at most finitely many $\zeta \in \mathbb{C}$ such that

$$
(\widehat{\phi}(\zeta+2 \pi k))_{k \in \mathbb{Z}}=\mathbf{0} .
$$

Furthermore, Ron proved in Theorem 1.1 of [19] that the shifts of a compactly supported distribution are linearly independent if and only if the Fourier-Laplace transform of $\phi$ satisfies

$$
(\widehat{\phi}(\zeta+2 \pi k))_{k \in \mathbb{Z}} \neq \mathbf{0} \quad \text { for all } \zeta \in \mathbb{C} .
$$

Comparing (1.8) and (1.9), we can immediately see that for a compactly supported function $\phi \in L_{2}(\mathbb{R})$, linear independence of the shifts of $\phi$ implies the stability of $\phi$. More recently, Jia and Wang characterized the linear independence of single variable refinable functions in terms of their masks.

It is easy to see from the definition of linear independence that when the function $\phi$ is a pseudo-spline of type I or II with order $(m, m-1)$ (which is the orthogonal refinable function for the first type or interpolatory refinable function for the second type), its shifts are linearly independent. It is also well known that a pseudo-spline of either type with order $(m, 0)$, which is a B-spline, and its shifts are linearly independent. It is very natural to ask whether an arbitrary pseudo-spline and its shifts are linearly independent. This is one of our motivations, but not the only one. The linear independence of a pseudo-spline $\phi$ and its shifts is a necessary and sufficient condition for the existence of a compactly supported dual refinable function $\phi^{d} \in L_{2}(\mathbb{R})$ of $\phi$. The proof of the necessity is simple. Recall that a compactly supported refinable function $\phi^{d} \in L_{2}(\mathbb{R})$ is a dual of $\phi$, if

$$
\left\langle\phi, \phi^{d}(\cdot-k)\right\rangle=\delta(k)
$$

holds for all $k \in \mathbb{Z}$ (see e.g. [5], [6]). Indeed, if there is a compactly supported function $\phi^{d} \in L_{2}(\mathbb{R})$ that is dual to $\phi$, then for $b \in \ell(\mathbb{Z})$ satisfying $\phi *^{\prime} b=0$, we have

$$
0=\left\langle\phi *^{\prime} b, \phi^{d}(\cdot-k)\right\rangle=\sum_{j \in \mathbb{Z}} b(j)\left\langle\phi(\cdot-j), \phi^{d}(\cdot-k)\right\rangle=b(k) \quad \text { for all } k \in \mathbb{Z} .
$$

However, the proof of the sufficiency is more complicated, and we refer to [17] and [18 for the details. It is well known that the existence of a compactly supported dual refinable function of a pseudo-spline is a key step in constructing a pair of biorthogonal wavelet systems from the given pseudo-spline.

Finally, we observe that since ${ }_{1} \widehat{a}_{(m, l)}(\xi)$ is a trigonometric polynomial with real coefficients, we have

$$
{ }_{2} \widehat{a}_{(m, l)}(\xi)=\left|{ }_{1} \widehat{a}_{(m, l)}(\xi)\right|^{2}={ }_{1} \widehat{a}_{(m, l)}(\xi) \cdot{ }_{1} \widehat{a}_{(m, l)}(-\xi) .
$$


This leads to

$$
{ }_{2} \widehat{\phi}_{(m, l)}(\xi)={ }_{1} \widehat{\phi}_{(m, l)}(\xi) \cdot{ }_{1} \widehat{\phi}_{(m, l)}(-\xi), \quad \xi \in \mathbb{R} .
$$

Since both ${ }_{1} \phi_{(m, l)}$ and ${ }_{2} \phi_{(m, l)}$ are compactly supported and bounded, their FourierLaplace transforms ${ }_{1} \widehat{\phi}_{(m, l)}(\zeta)$ and ${ }_{2} \widehat{\phi}_{(m, l)}(\zeta)$ are analytic on $\mathbb{C}$. Hence, (1.11) holds for all $\zeta \in \mathbb{C}$, i.e.

$$
{ }_{2} \widehat{\phi}_{(m, l)}(\zeta)={ }_{1} \widehat{\phi}_{(m, l)}(\zeta) \cdot{ }_{1} \widehat{\phi}_{(m, l)}(-\zeta), \quad \zeta \in \mathbb{C} .
$$

The identity (1.12) implies that the set of all zeros of ${ }_{1} \widehat{\phi}_{(m, l)}(\zeta)$ is contained in that of ${ }_{2} \widehat{\phi}_{(m, l)}(\zeta)$ for $\zeta \in \mathbb{C}$.

Applying (1.9), we conclude the following proposition.

Proposition 1.1. Assume that the shifts of pseudo-spline ${ }_{2} \phi_{(m, l)}$ of type II with order $(m, l)$ are linearly independent. Then the shifts of pseudo-spline ${ }_{1} \phi_{(m, l)}$ of type I with the same order are linearly independent.

In the rest of the paper, we will focus on the verification of the linear independence of the shifts of pseudo-splines of type II.

\section{LINEAR INDEPENDENCE}

This section is to verify the linear independence of the shifts of pseudo-splines. We start with two lemmata. The first lemma is implied by Theorem 1 and 2 of a paper of Jia and Wang (see [16]). Instead of stating both theorems of [16] and deducing the following lemma by using them, here we include a direct proof which is essentially derived from Jia and Wang's proof of Theorem 1 and 2 in [16].

We say that a Laurent polynomial $\tilde{a}$ has symmetric zeros on $\mathbb{C} \backslash\{0\}$ if there is a $z_{0} \in \mathbb{C} \backslash\{0\}$ such that

$$
\tilde{a}\left(z_{0}\right)=\tilde{a}\left(-z_{0}\right)=0 .
$$

Lemma 2.1. Let $\phi \in L_{2}(\mathbb{R})$ be a compactly supported refinable function with (finitely supported) refinement mask $a$. The shifts of $\phi$ are linearly independent if and only if:

(1) $\phi$ is stable;

(2) the symbol $\tilde{a}$ does not have any symmetric zeros on $\mathbb{C} \backslash\{0\}$.

Proof. We first show the necessity of (1) and (2). Condition (1) is necessary for the linear independence of the shifts of $\phi$ implied by (1.8) and (1.9). The necessity of (2) is proven by contradiction. Suppose there exists $z_{0}=e^{-i \zeta_{0}} \in \mathbb{C} \backslash\{0\}$ such that $\tilde{a}\left(e^{-i \zeta_{0}}\right)=\tilde{a}\left(-e^{-i \zeta_{0}}\right)=0$. Applying the Fourier-Laplace transform given in (1.2), one obtains, for any $k \in \mathbb{Z}$,

$$
\widehat{\phi}\left(2 \zeta_{0}+4 k \pi\right)=\widehat{\phi}\left(\zeta_{0}+2 k \pi\right) \tilde{a}\left(e^{-i \zeta_{0}}\right)=0
$$

and

$$
\widehat{\phi}\left(2 \zeta_{0}+(4 k+2) \pi\right)=\widehat{\phi}\left(\zeta_{0}+2 k \pi+\pi\right) \tilde{a}\left(-e^{-i \zeta_{0}}\right)=0 .
$$

These two identities imply that $\widehat{\phi}\left(2 \zeta_{0}+2 k \pi\right)=0$ for all $k \in \mathbb{Z}$, which contradicts the linear independence of the shifts of $\phi$ by (1.9).

Next, we show the sufficiency of (1) and (2), which is again shown by contradiction. Suppose that $\phi$ and its shifts are not linearly independent. Then, there 
is a $\zeta_{0} \in \mathbb{C}$, such that $\widehat{\phi}\left(\zeta_{0}+2 k \pi\right)=0$ for all $k \in \mathbb{Z}$. Since $(\widehat{\phi}(2 k \pi))_{k \in \mathbb{Z}} \neq \mathbf{0}$, $\zeta_{0} \in \mathbb{C} \backslash\{0\}$. Applying (1.2) again, one obtains, for any $k \in \mathbb{Z}$,

$$
0=\widehat{\phi}\left(\zeta_{0}+4 k \pi\right)=\widehat{\phi}\left(\zeta_{0} / 2+2 k \pi\right) \tilde{a}\left(e^{-i \zeta_{0} / 2}\right)
$$

and

$$
0=\widehat{\phi}\left(\zeta_{0}+(4 k+2) \pi\right)=\widehat{\phi}\left(\zeta_{0} / 2+\pi+2 k \pi\right) \tilde{a}\left(-e^{-i \zeta_{0} / 2}\right) .
$$

Since $\tilde{a}(z)$ does not have symmetric zeros on $\mathbb{C} \backslash\{0\}$, we conclude that at least one of the two sets of identities $\widehat{\phi}\left(\zeta_{0} / 2+2 k \pi\right)=0, k \in \mathbb{Z}$ and $\widehat{\phi}\left(\zeta_{0} / 2+\pi+2 k \pi\right)=0$, $k \in \mathbb{Z}$ holds. Let $\zeta_{1}=\zeta_{0} / 2$ or $\zeta_{1}=\zeta_{0} / 2+\pi$. (Here the choice depends on whether $\widehat{\phi}\left(\zeta_{0} / 2+2 k \pi\right)=0, k \in \mathbb{Z}$ or $\widehat{\phi}\left(\zeta_{0} / 2+\pi+2 k \pi\right)=0, k \in \mathbb{Z}$.) Repeating this process, one obtains $\zeta_{2}=\zeta_{1} / 2$ or $\zeta_{2}=\zeta_{1} / 2+\pi$. Continuing the process, one obtains a set of numbers $A:=\left\{\zeta_{0}, \zeta_{1}, \zeta_{2}, \cdots\right\}$ such that $\left(\widehat{\phi}\left(\zeta_{j}+2 k \pi\right)\right)_{k \in \mathbb{Z}}=\mathbf{0}$ for $j=0,1,2, \cdots$. However, by Proposition 2.1 of Ron in [19, the set $A$ must be finite (also see [16]). Hence, there must exist some integers $0 \leq p<q$, such that $\zeta_{p}=\zeta_{q}$. Since $\zeta_{p}=\frac{\zeta_{0}}{2^{p}}+r \pi, \zeta_{q}=\frac{\zeta_{0}}{2^{q}}+s \pi$ for some rational number $r$ and $s$, we have

$$
\frac{\zeta_{0}}{2^{p}}+r \pi=\frac{\zeta_{0}}{2^{q}}+s \pi
$$

This leads to the fact that $\zeta_{0}$ is a real number which implies that

$$
\left(\widehat{\phi}\left(\zeta_{0}+2 k \pi\right)\right)_{k \in \mathbb{Z}}=\mathbf{0} \quad \text { with } \zeta_{0} \in \mathbb{R} .
$$

This contradicts the stability of $\phi$ (which is (1)) by (1.8).

Lemma 2.1 says that, in order to show the linear independence of the shifts of pseudo-splines of type II, we need to verify: (i) pseudo-splines of type II are stable; (ii) the symbol of an arbitrary pseudo-spline of type II does not have any symmetric zeros on $\mathbb{C} \backslash\{0\}$. The stability of pseudo-splines of type II follows from the definitions of pseudo-splines. Here, we give a short proof for the sake of completeness.

Lemma 2.2. Pseudo-splines of type II are stable.

Proof. Since pseudo-splines are compactly supported and belong to $L_{2}(\mathbb{R})$ (see e.g. Proposition 3.5 of [11]), the stability of them is equivalent to (1.8). Let $\phi_{(m, l)}$ be the pseudo-spline of type II with order $(m, l)$ and let $\widehat{a}_{(m, l)}$ be its refinement mask. By Definition (1.4), for each fixed $m \geq 1$ and for every $0 \leq l \leq m-1$, the inequality

$$
\cos ^{2 m}(\xi / 2) \leq \widehat{a}_{(m, l)}(\xi)
$$

holds for all $\xi \in \mathbb{R}$. Therefore, by (1.5), we have for all $\xi \in \mathbb{R}$,

$$
\left|\widehat{B}_{2 m}(\xi)\right| \leq\left|\widehat{\phi}_{(m, l)}(\xi)\right| .
$$

Since $B_{2 m}$ is stable, the vector $\left(\widehat{B}_{2 m}(\xi+2 k \pi)\right)_{k \in \mathbb{Z}} \neq \mathbf{0}$ for every $\xi \in \mathbb{R}$. Hence, $\left(\widehat{\phi}_{(m, l)}(\xi+2 k \pi)\right)_{k \in \mathbb{Z}} \neq \mathbf{0}$ for every $\xi \in \mathbb{R}$, which is equivalent to the fact that $\phi_{(m, l)}$ is stable.

By Lemmas 2.1 and 2.2. to show that the shifts of a pseudo-spline of type II are linearly independent, we only need to show that the symbol of it has no symmetric zeros on $\mathbb{C} \backslash\{0\}$. Now we compute the symbols of pseudo-splines of type II. Recall 
that the refinement mask of a pseudo-spline of type II with order $(m, l)$ is given by (1.4), i.e.

$$
{ }_{2} \widehat{a}(\xi)=\cos ^{2 m}(\xi / 2) \sum_{j=0}^{l}\left(\begin{array}{c}
m+l \\
j
\end{array}\right) \sin ^{2 j}(\xi / 2) \cos ^{2(l-j)}(\xi / 2) .
$$

Using

$$
\cos ^{2}(\xi / 2)=\frac{1+\cos (\xi)}{2}=\frac{2+e^{i \xi}+e^{-i \xi}}{4}=\frac{\left(1+e^{-i \xi}\right)^{2}}{4 e^{-i \xi}}
$$

and

$$
\sin ^{2}(\xi / 2)=\frac{1-\cos (\xi)}{2}=\frac{2-e^{i \xi}-e^{-i \xi}}{4}=\frac{-\left(1-e^{-i \xi}\right)^{2}}{4 e^{-i \xi}},
$$

one obtains

${ }_{2} \widehat{a}(\xi):=\frac{\left(1+e^{-i \xi}\right)^{2 m}}{\left(4 e^{-i \xi}\right)^{m}} \sum_{j=0}^{l}\left(\begin{array}{c}m+l \\ j\end{array}\right)\left(\frac{-\left(1-e^{-i \xi}\right)^{2}}{4 e^{-i \xi}}\right)^{j}\left(\frac{\left(1+e^{-i \xi}\right)^{2}}{4 e^{-i \xi}}\right)^{l-j}, \quad$ for $\xi \in \mathbb{R}$.

Extending the above trigonometric polynomial to the Laurent polynomial, one obtains the symbol of the pseudo-spline of type II with order $(m, l)$ :

$$
{ }_{2} \tilde{a}(z):=\frac{(1+z)^{2 m}}{(4 z)^{m}} \sum_{j=0}^{l}\left(\begin{array}{c}
m+l \\
j
\end{array}\right)\left(\frac{-(1-z)^{2}}{4 z}\right)^{j}\left(\frac{(1+z)^{2}}{4 z}\right)^{l-j}, \quad z \in \mathbb{C} \backslash\{0\} .
$$

Before proving the main theorem of this paper, we need to give the following proposition first. The proof of it employs Rouché's theorem (see e.g. [2]), which states: Suppose two functions $f(z)$ and $g(z)$ are analytic inside and on a simple closed contour $C$, and suppose

$$
|f(z)|>|g(z)| \text { for all } z \in C \text {. }
$$

Then $f$ and $f+g$ have the same number of zeros, counting multiplicities, inside $C$.

Proposition 2.3. Let

$$
P(z)=\sum_{j=0}^{l} c_{j} z^{j}
$$

be a polynomial with real coefficients satisfying

$$
c_{l}>c_{l-1}>\cdots>c_{0}>0
$$

Then, all zeros of $P(z)$ are contained in the open unit disk $D:=\{z \in \mathbb{C}:|z|<1\}$.

Proof. Let

$$
\rho:=\max _{1 \leq j \leq l}\left\{\frac{c_{j-1}}{c_{j}}\right\} .
$$

Since $c_{j}$ is strictly greater than $c_{j-1}, 0<\rho<1$ and $\rho c_{j} \geq c_{j-1}$ for all $1 \leq j \leq l$. Consider

$$
Q(z):=(\rho-z) P(z) .
$$

Then,

$$
\begin{aligned}
Q(z) & =\rho P(z)-z P(z) \\
& =\rho c_{0}+\left(\rho c_{1}-c_{0}\right) z+\left(\rho c_{2}-c_{1}\right) z^{2}+\cdots+\left(\rho c_{l}-c_{l-1}\right) z^{l}-c_{l} z^{l+1} \\
& =g(z)+f(z)
\end{aligned}
$$


where

$f(z):=-c_{l} z^{l+1} \quad$ and $\quad g(z):=\rho c_{0}+\left(\rho c_{1}-c_{0}\right) z+\left(\rho c_{2}-c_{1}\right) z^{2}+\cdots+\left(\rho c_{l}-c_{l-1}\right) z^{l}$.

Note that when $|z|=1$, we have

$$
\begin{aligned}
|g(z)| & \leq \rho c_{0}+\left(\rho c_{1}-c_{0}\right)+\left(\rho c_{2}-c_{1}\right)+\cdots+\left(\rho c_{l}-c_{l-1}\right) \\
& =(\rho-1) c_{0}+(\rho-1) c_{1}+\cdots+(\rho-1) c_{l-1}+\rho c_{l} \\
& =\rho c_{l}-(1-\rho) \sum_{j=0}^{l-1} c_{j} \\
& <c_{l}=\left|-c_{l} z^{l+1}\right|=|f(z)| .
\end{aligned}
$$

Since $f$ and $g$ are analytic on $\{z \in \mathbb{C}:|z| \leq 1\}$, Rouché's theorem asserts that $Q=f+g$ has the same number of zeros as that of $f$ in $D=\{z \in \mathbb{C}:|z|<1\}$. Since $f$ has $l+1$ zeros in $D, Q$ must have exactly $l+1$ zeros in $D$. Since $Q$ has only $l+1$ zeros and since zeros of $P$ are a subset of the zeros of $Q$, all zeros of $P$ must be in $D$.

Next, we prove the main theorem of this paper.

Theorem 2.4. The shifts of a pseudo-spline of type II are linearly independent.

Proof. Since Lemma 2.2 shows that pseudo-splines of type II with arbitrary orders are stable, in order to prove the linear independence of the shifts of a given pseudospline of type II, one only needs to show that the $\operatorname{symbol}_{2} \tilde{a}(z)$ of it has no symmetric zeros on $\mathbb{C} \backslash\{0\}$, by Lemma 2.1.

The symbol ${ }_{2} \tilde{a}(z)$ given by (2.5) can be rewritten as

$$
\begin{aligned}
{ }_{2} \tilde{a}(z) & =\frac{(1+z)^{2 m}}{(4 z)^{m}} \sum_{j=0}^{l}\left(\begin{array}{c}
m+l \\
j
\end{array}\right)\left(\frac{-(1-z)^{2}}{4 z}\right)^{j}\left(\frac{(1+z)^{2}}{4 z}\right)^{l-j} \\
& =\frac{(1+z)^{2 m}}{(4 z)^{m+l}} \sum_{j=0}^{l}\left(\begin{array}{c}
m+l \\
j
\end{array}\right)\left(-(1-z)^{2}\right)^{j}(1+z)^{2(l-j)} \\
& =\frac{(1+z)^{2 m+2 l}}{(4 z)^{m+l}} \sum_{j=0}^{l}\left(\begin{array}{c}
m+l \\
j
\end{array}\right)\left(\frac{-(1-z)^{2}}{(1+z)^{2}}\right)^{j} .
\end{aligned}
$$

Since $z=-1$ is a zero of ${ }_{2} \tilde{a}(z)$, while ${ }_{2} \tilde{a}(z)=1$ when $z=1,{ }_{2} \tilde{a}(z)$ having no symmetric zeros on $\mathbb{C} \backslash\{0\}$ is equivalent to

$$
h(z):=\sum_{j=0}^{l}\left(\begin{array}{c}
m+l \\
j
\end{array}\right)\left(\frac{-(1-z)^{2}}{(1+z)^{2}}\right)^{j},
$$

having no symmetric zeros on $\mathbb{C} \backslash\{0,1,-1\}$.

Consider

$$
P(x)=\sum_{j=0}^{l} b_{j} x^{j}, \quad \text { with } b_{j}=\left(\begin{array}{c}
m+l \\
j
\end{array}\right), x \in \mathbb{C} .
$$

We first show that Proposition 2.3 can be applied to $P$ to conclude that the zeros of $P$ lie inside of the unit disk of $\mathbb{C}$. For this, we need to show that for given $m>0,0 \leq l \leq m-1$,

$$
b_{j+1}>b_{j}>0, \quad 0 \leq j \leq l-1 .
$$


Note that

$$
\left(\begin{array}{c}
m+l \\
j+1
\end{array}\right)=\frac{m+l-j}{j+1}\left(\begin{array}{c}
m+l \\
j
\end{array}\right)
$$

Since $j \leq l-1$, replacing $j$ by $l-1$ in $\frac{m+l-j}{j+1}$, the right-hand side of the above identity decreases and becomes

$$
\frac{m+1}{l}\left(\begin{array}{c}
m+l \\
j
\end{array}\right)
$$

which is larger than $\left(\begin{array}{c}m+l \\ j\end{array}\right)$ by $l \leq m-1$. This shows that $b_{j+1}>b_{j}, j=0, \ldots, l-1$. It is clear that $b_{0}=1>0$. With (2.7), applying Proposition 2.3, one concludes that all zeros of $P(x)$ must be in $\{x \in \mathbb{C}:|x|<1\}$. Let $z_{0}$ be an arbitrary zero of $h$ in $\mathbb{C} \backslash\{0,1,-1\}$. Then, the above conclusion on the zeros of $P$ implies that $z_{0}$ must satisfy

$$
\left|\frac{-\left(1-z_{0}\right)^{2}}{\left(1+z_{0}\right)^{2}}\right|<1
$$

Suppose $h$ has symmetric zeros $z_{0}$ and $-z_{0}$. Then, $-z_{0}$ must also satisfy

$$
\left|\frac{-\left(1+z_{0}\right)^{2}}{\left(1-z_{0}\right)^{2}}\right|<1
$$

Since

we conclude that

$$
\left|\frac{-\left(1-z_{0}\right)^{2}}{\left(1+z_{0}\right)^{2}}\right|=\frac{1}{\left|\frac{-\left(1+z_{0}\right)^{2}}{\left(1-z_{0}\right)^{2}}\right|}
$$

$$
\left|\frac{-\left(1-z_{0}\right)^{2}}{\left(1+z_{0}\right)^{2}}\right|>1
$$

which contradicts (2.8). This leads to the fact that $h$ has no symmetric zeros on $\mathbb{C} \backslash\{0,1,-1\}$, and hence, ${ }_{2} \tilde{a}(z)$ has no symmetric zeros on $\mathbb{C} \backslash\{0\}$. This, together with the stability of pseudo-splines of type II, proves the linear independence of the shifts of an arbitrary given pseudo-spline of type II by Lemma 2.1.

\section{ACKNOWLEDGMENTS}

The authors thank the anonymous referee for his suggestions that led to Proposition 1.1

\section{REFERENCES}

1. C. de Boor, A Practical Guide to Splines, Springer-Verlag, New York, 1978. MR0507062 (80a:65027)

2. J. W. Brown and R. V. Churchill, Complex Variables and Applications, 7th ed., McGraw-Hill Higher Education, Boston, 2004. MR0730937 (84k:30002)

3. C. de Boor, R. DeVore and A. Ron, The structure of finitely generated shift-invariant spaces in $L_{2}\left(\mathbb{R}^{d}\right)$, J. Funct. Anal. 119 (1994), 37-78. MR.1255273 (95g:46050)

4. A. Ben-Artzi and A. Ron, On the integer translates of a compactly supported function: dual bases and linear projectors, SIAM J. Math. Anal. 21 (1990), 1550-1562. MR1075591 (91j:41009)

5. A. Cohen and I. Daubechies, A stability criterion for biorthogonal wavelet bases and their related subband coding scheme, Duke Math. J. 68 (2) (1992), 313-335. MR.1191564|(94b:94005)

6. A. Cohen, I. Daubechies and J. C. Feauveau, Biorthogonal bases of compactly supported wavelets, Comm. Pure Appl. Math. 45 (1992), 485-560. MR.1162365 (93e:42044) 
7. I. Daubechies, Ten Lectures on Wavelets, in: CBMS Conf. Series in Appl. Math., vol. 61, SIAM, Philadelphia, 1992. MR.1162107 (93e:42045)

8. I. Daubechies, B. Han, A. Ron, and Z. Shen, Framelets: MRA-based constructions of wavelet frames, Appl. Comput. Harmon. Anal. 14 (1) (2003), 1-46. MR1971300 (2004a:42046)

9. W. Dahmen and C. A. Micchelli, Translates of multivariate splines, Linear Algebra Appl. 52 (1983), 217-234. MR0709352 (85e:41033)

10. W. Dahmen and C. A. Micchelli, On the local linear independence of translates of a box spline, Studia Math. 82 (1985), 243-262. MR0825481 (87k:41008)

11. B. Dong and Z. Shen, Pseudo-splines, wavelets and framelets, preprint (2004).

12. S. Dubuc, Interpolation through an iterative scheme, J. Math. Anal. Appl. 114 (1986), 185204. MR0829123 (88b:41003)

13. R. Q. Jia, Linear independence of translates of a box spline, J. Approx. Theory 40 (1984), 158-160. MR0732698 (85h:41025)

14. R. Q. Jia, Local linear independence of the translates of a box spline, Constr. Approx. 1 (1985), 175-182. MR0891538(88d:41017)

15. R. Q. Jia and C. A. Micchelli, Using the refinement equations for the construction of prewavelets. II. Powers of two, Curves and Surfaces, 209-246, Academic Press, Boston, MA, 1991. MR 1123739 (93e:65024)

16. R. Q. Jia and J. Z. Wang, Stability and linear independence associated with wavelet decompositions, Proc. Am. Math. Soc. 117 (4) (1993), 1115-1124. MR.1120507 (93e:42046)

17. P. G. Lemarié-Rieusset, On the existence of compactly supported dual wavelets, Appl. Comput. Harmon. Anal. 3 (1997), 117-118. MR.1429683 (97h:42018)

18. P. G. Lemarié-Rieusset, Fonctions d'échelle interpolantes, polynômes de Bernstein et ondelettes non stationnaires, Rev. Mat. Iberoamericana 13 (1) (1997), 91-188. MR 1462330 (98k:42045)

19. A. Ron, A necessary and sufficient condition for the linear independence of the integer translates of a compactly supported distribution, Constr. Approx. 5 (1989), 297-308. MR0996932 (90g:41019)

20. A. Ron and Z. Shen, Frames and stable bases for shift-invariant subspaces of $L_{2}\left(\mathbb{R}^{d}\right)$, Canad. Math. J. 47 (1995), 1051-1094. MR1350650 (96k:42049)

21. A. Ron and Z. Shen, Affine systems in $L_{2}\left(\mathbb{R}^{d}\right)$ : the analysis of the analysis operator, J. Funct. Anal. 148 (2) (1997), 408-447. MR.1469348 (99g:42043)

Department of Mathematics, National University of Singapore, Science Drive 2, SingaPORE 117543 , SingaPORE

Current address: Department of Mathematics, University of California, Los Angeles, Box 951555, Los Angeles, California 90095-1555

E-mail address: g0301173@nus.edu.sg; bdong@math.ucla.edu

Department of Mathematics, National University of Singapore, Science Drive 2, Singapore 117543, Singapore

E-mail address: matzuows@nus.edu.sg 